\title{
Universal estimate of the gradient for parabolic equations
}

\author{
Nikolai Dokuchaev \\ Department of Mathematics, Trent University, Ontario, Canada
}

\begin{abstract}
We suggest a modification of the estimate for weighted Sobolev norms of solutions of parabolic equations such that the matrix of the higher order coefficients is included into the weight for the gradient. More precisely, we found the upper limit estimate that can be achieved by variations of the zero order coefficient. As an example of applications, an asymptotic estimate was obtained for the gradient at initial time. The constant in the estimates is the same for all possible choices of the dimension, domain, time horizon, and the coefficients of the parabolic equation. As an another example of application, existence and regularity results are obtained for parabolic equations with time delay for the gradient.
\end{abstract}

AMS 2000 subject classification: 35K10, 35K15, 35K20

Key words and phrases: parabolic equations, regularity, solution gradient, asymptotic estimates, parabolic delay equations.

\section{Introduction}

We study Dirichlet boundary value problems for linear parabolic equations. The classical results give estimates for Sobolev norms of the solution via the $L_{2}$-norm of the nonhomogeneous term (see, e.g., the second energy inequality (4.56) in Ladyzhenskaia (1985), Chapter III). We suggest a modification of this estimate for weighted Sobolev norms such that the matrix of the higher order coefficients is included into the weight for the gradient. We found the limit upper estimate that can be achieved by variations of the zero order coefficient. More precisely, we obtain estimates for $e^{-K t} u(x, t)$ via $L_{2}$-norm of $e^{-K t} h(x, t)$, where $u(x, t)$ and $h(x, t)$ is the solution and the nonhomogeneous term respectively, and where $K>0$ is being variable. As an example of applications, an asymptotic upper estimate was obtained for a weighted $L_{2}$-norm of the gradient at initial time. The constants in these estimates are the same for all possible choices of the dimension, domain, time horizon, and the coefficients of the parabolic equations, i.e., these 
estimates can be called universal estimates. As an another example of applications, we establish solvability and regularity for special parabolic equations such that the gradient is included with time delay.

\section{Definitions}

\section{Spaces and classes of functions.}

We denote by $|\cdot|$ the Euclidean norm in $\mathbf{R}^{k}$ and the Frobenius norm in $\mathbf{R}^{k \times m}$, and we denote by $\|\cdot\|_{X}$ the norm in a linear normed space $X$. We denote by $(\cdot, \cdot)_{X}$ the scalar product in a Hilbert space $X$. For a Banach space $X$, we denote by $C([a, b], X)$ the Banach space of continuous functions $x:[a, b] \rightarrow X$.

We are given an open domain $D \subseteq \mathbf{R}^{n}$ such that either $D=\mathbf{R}^{n}$ or $D$ is bounded with $C^{2}$-smooth boundary $\partial D$.

Let $T>0$ be given, and let $Q \triangleq D \times(0, T)$.

We denote by $W_{2}^{m}(D)$ the Sobolev space of functions that belong to $L_{2}(D)$ together with the distributional derivatives up to the $m$ th order, $m \geq 0$.

Let $H^{0} \triangleq L_{2}(D)$, and let $H^{1} \triangleq \stackrel{0}{=} W_{2}^{1}(D)$ be the closure in the $W_{2}^{1}(D)$-norm of the set of all smooth functions $u: D \rightarrow \mathbf{R}$ such that $\left.u\right|_{\partial D} \equiv 0$. Let $H^{2}=W_{2}^{2}(D) \cap H^{1}$ be the space equipped with the norm of $W_{2}^{2}(D)$. The spaces $H^{k}$ are Hilbert spaces, and $H^{k}$ is a closed subspace of $W_{2}^{k}(D), k=1,2$.

Let $H^{-1}$ be the dual space to $H^{1}$, with the norm $\|\cdot\|_{H^{-1}}$ such that if $u \in H^{0}$ then $\|u\|_{H^{-1}}$ is the supremum of $(u, w)_{H^{0}}$ over all $w \in H^{1}$ such that $\|w\|_{H^{1}} \leq 1 . H^{-1}$ is a Hilbert space.

We will write $(u, w)_{H^{0}}$ for $u \in H^{-1}$ and $w \in H^{1}$, meaning the obvious extension of the bilinear form from $u \in H^{0}$ and $w \in H^{1}$.

We denote by $\bar{\ell}_{1}$ the Lebesgue measure in $\mathbf{R}$, and we denote by $\overline{\mathcal{B}}_{1}$ the $\sigma$-algebra of Lebesgue sets in $\mathbf{R}^{1}$.

For $k=-1,0,1,2$, we introduce the spaces

$$
X^{k} \triangleq L_{2}\left([0, T], \overline{\mathcal{B}}_{1}, \bar{\ell}_{1} ; H^{k}\right), \quad \mathcal{C}^{k} \triangleq C\left([0, T] ; H^{k}\right)
$$

We introduce the spaces

$$
Y^{k} \triangleq X^{k} \cap \mathcal{C}^{k-1}, \quad k \geq 0
$$

with the norm $\|u\|_{Y^{k}} \triangleq\|u\|_{X^{k}}+\|u\|_{\mathcal{C}^{k-1}}$. 
We use the notations

$$
\nabla u \triangleq\left(\frac{\partial u}{\partial x_{1}}, \frac{\partial u}{\partial x_{2}}, \ldots, \frac{\partial u}{\partial x_{n}}\right)^{\top}, \quad \nabla \cdot U=\sum_{i=1}^{n} \frac{\partial U_{i}}{\partial x_{i}}
$$

for functions $u: \mathbf{R}^{n} \rightarrow \mathbf{R}$ and $U=\left(U_{1}, \ldots, U_{n}\right)^{\top}: \mathbf{R}^{n} \rightarrow \mathbf{R}^{n}$. In addition, we use the notation

$$
(U, V)_{H^{0}}=\sum_{i=1}^{n}\left(U_{i}, V_{i}\right)_{H^{0}}, \quad\|U\|_{H^{0}}=(U, U)_{H^{0}}^{1 / 2}
$$

for functions $U, V: D \rightarrow \mathbf{R}^{n}$, where $U=\left(U_{1}, \ldots, U_{n}\right)$ and $V=\left(V_{1}, \ldots, V_{n}\right)$.

\section{The boundary value problem}

We consider the problem

$$
\begin{aligned}
& \frac{\partial u}{\partial t}=\mathcal{A} u+h, \quad t \in(0, T), \\
& u(x, 0)=0,\left.\quad u(x, t)\right|_{x \in \partial D}=0 .
\end{aligned}
$$

Here $u=u(x, t),(x, t) \in Q, h \in L_{2}(Q)$, and

$$
\mathcal{A} y \triangleq \sum_{i=1}^{n} \frac{\partial}{\partial x_{i}} \sum_{j=1}^{n}\left(b_{i j}(x, t) \frac{\partial y}{\partial x_{j}}(x)\right)+\sum_{i=1}^{n} f_{i}(x, t) \frac{\partial y}{\partial x_{i}}(x)+\lambda(x, t) y(x),
$$

where $b(x, t): \mathbf{R}^{n} \times[0, T] \rightarrow \mathbf{R}^{n \times n}, f(x, t): \mathbf{R}^{n} \times[0, T] \rightarrow \mathbf{R}^{n}$, and $\lambda(x, t): \mathbf{R}^{n} \times[0, T] \rightarrow \mathbf{R}$, are bounded measurable functions, and where $b_{i j}, f_{i}, x_{i}$ are the components of $b, f$, and $x$. The matrix $b=b^{\top}$ is symmetric.

To proceed further, we assume that Conditions 1 12 remain in force throughout this paper.

Condition 1 There exists a constant $\delta>0$ such that

$$
\xi^{\top} b(x, t) \xi \geq \delta|\xi|^{2} \quad \forall \xi \in \mathbf{R}^{n},(x, t) \in Q
$$

Condition 2 The functions $b(x, t): \mathbf{R}^{n} \times \mathbf{R} \rightarrow \mathbf{R}^{n \times n}, f(x, t): \mathbf{R}^{n} \times \mathbf{R} \rightarrow \mathbf{R}^{n}, \lambda(x, t): \mathbf{R}^{n} \times$ $\mathbf{R} \rightarrow \mathbf{R}$, are differentiable in $x$, the function $b(x, t)$ is differentiable in $t$, and the corresponding derivatives are bounded.

We introduce the sets of parameters

$$
\begin{aligned}
\mu \triangleq(T, n, D, b(\cdot), f(\cdot), \lambda(\cdot)), & \\
\mathcal{P}=\mathcal{P}(\mu) \triangleq(T, n, D, \delta, & \underset{(x, t) \in Q}{\operatorname{ess} \sup }\left(|b(x, t)|+|f(x, t)|+|\lambda(x, t)|+\left|\frac{\partial b}{\partial x}(x, t)\right|+\left|\frac{\partial f}{\partial x}(x, t)\right|\right. \\
& \left.\left.+\left|\frac{\partial \lambda}{\partial x}(x, t)\right|+\left|\frac{\partial b}{\partial t}(x, t)\right|\right)\right) .
\end{aligned}
$$




\section{Special estimates for the solution}

By the classical solvability results, there exists a unique solution $u \in Y^{1}$ of problem (1) for any $h \in L_{2}(Q)$ (see, e.g., Ladyzhenskaia (1985), Chapter III, $\left.\S \S 4-5\right)$. Moreover, the second energy inequality gives that, for any $K \in \mathbf{R}$ and $M \geq 0$, there exists a constant $\tilde{C}(K, M, \mathcal{P})>0$ such that

$$
\begin{aligned}
& e^{-2 K t}(\nabla u(\cdot, t), b(\cdot, t) \nabla u(\cdot, t))_{H^{0}} \\
& +M\left[e^{-2 K t}\|u(\cdot, t)\|_{H^{0}}^{2}+\int_{0}^{t} e^{-2 K s}(\nabla u(\cdot, s), b(\cdot, s) \nabla u(\cdot, s))_{H^{0}} d s\right] \\
& \leq \tilde{C}(K, M, \mathcal{P}) \int_{0}^{t} e^{-2 K s}\|h(\cdot, s)\|_{H^{0}}^{2} d s \quad \forall h \in L_{2}(Q), t \in(0, T],
\end{aligned}
$$

for any solution $u$ of problem (11). This estimate follows immediately from inequality (4.56) from Ladyzhenskaia (1985), Chapter III, and from obvious estimates

$$
e^{-2 K t}(\nabla w, b(\cdot, t) \nabla w)_{H^{0}} \leq c_{1}\|w\|_{H^{1}}^{2}, \quad\|w\|_{H^{0}}^{2} \leq c_{2} e^{-2 K t}\|w\|_{H^{0}}^{2} \quad \forall t \in[0, T],
$$

for any $w \in H^{1}$ and $w \in H^{0}$ respectively, where $c_{1} \triangleq \operatorname{ess}_{\sup }|b(x, t)|$ and $c_{2} \triangleq e^{2 K T}$.

Let $C(K, M, \mathcal{P}) \triangleq \inf \tilde{C}(K, M, \mathcal{P})$, where the infimum is taken over all $\tilde{C}(K, M, \mathcal{P})$ such that (41) holds.

\section{Theorem 1}

$$
\sup _{\mu, M \geq 0} \inf _{K \geq 0} C(K, M, \mathcal{P}(\mu)) \leq \frac{1}{2}
$$

Corollary 1 For any $\mu$ and $\varepsilon>0$, there exists $K=K(\varepsilon, \mathcal{P}(\mu)) \geq 0$ such that

$$
\begin{array}{r}
\sup _{s \in[0, t]} e^{-2 K s}(\nabla u(\cdot, s), b(\cdot, s) \nabla u(\cdot, s))_{H^{0}} \leq\left(\frac{1}{2}+\varepsilon\right) \int_{0}^{t} e^{-2 K s}\|h(\cdot, s)\|_{H^{0}}^{2} d s \\
\forall h \in L_{2}(Q), t \in(0, T]
\end{array}
$$

where $u$ is the solution of problem (1) for the corresponding $h$.

Remark 1 For the case of a non-divergent operator $\mathcal{A} u=b_{i j} u_{x_{i} x_{j}}+f_{i} u_{x_{i}}+\lambda u$, the differentiability of the coefficients in Condition 2 can be weakened using the approach from Dokuchaev (2005). 


\section{Applications: asymptotic estimate at initial time}

Let

$$
X_{c}^{0} \triangleq\left\{h \in X^{0}: \lim _{t \rightarrow 0+} \frac{1}{t} \int_{0}^{t}\|h(\cdot, s)\|_{H^{0}}^{2} d s=\|h(\cdot, 0)\|_{H^{0}}^{2}\right\} .
$$

The condition that $h \in X_{c}^{0}$ is not restrictive for $h \in X^{0}$; for instance, it holds if $t=0$ is a Lebesgue point for $\|h(\cdot, t)\|_{H^{0}}^{2}$.

Theorem 2 For any admissible set of parameters $\mu$,

$$
\varlimsup_{t \rightarrow 0+} \sup _{h \in X_{c}^{0}} \frac{1}{t} \frac{(\nabla u(\cdot, t), b(\cdot, t) \nabla u(\cdot, t))_{H^{0}}}{\|h(\cdot, 0)\|_{H^{0}}^{2}} \leq \frac{1}{2},
$$

where $u$ is the solution of problem (1) for the corresponding $h$.

\section{On the sharpness of the estimates}

Theorem 3 There exists a set of parameters $(n, D, b(\cdot), f(\cdot), \lambda(\cdot))$ such that, for any $T>0$, $M \geq 0$,

$$
\inf _{K \geq 0} C(K, M, \mathcal{P}(\mu))=\frac{1}{2}
$$

for $\mu=(T, n, D, b(\cdot), f(\cdot), \lambda(\cdot))$.

Theorem 4 There exists a set of parameters $(n, D, b(\cdot), f(\cdot), \lambda(\cdot))$ such that

$$
\varlimsup_{t \rightarrow 0+} \sup _{h \in X_{c}^{0}} \frac{1}{t} \frac{(\nabla u(\cdot, t), b(\cdot, t) \nabla u(\cdot, t))_{H^{0}}}{\|h(\cdot, 0)\|_{H^{0}}^{2}}=\frac{1}{2},
$$

where $u$ is the solution of problem (1) for the corresponding $h$.

\section{Applications: parabolic equations with time delay}

Theorem 1 can be also applied to analysis of parabolic equations with time delay. These equations have many applications, and they were intensively studied, including equations with delay operators of a general form defined on the path of past values (see, e.g., Bátkai and Piazzera (2001), Pao (1997), Poorkarimi and Wiener (1999), Stein et al (2005), and references here). We use Theorem 1 to obtain sufficient conditions of solvability in $Y^{2}$ for the special case when the first derivatives of solutions are affected by time delay represented by a general measurable function. As far as we know, this case was not covered in the existing literature. 
Let $\tau(\cdot):[0, T] \rightarrow \mathbf{R}$ be a given measurable function such that $\tau(t) \in[0, t]$. Non-monotonic, piecewise constant, or nowhere continuous functions $\tau(\cdot)$ are not excluded.

For functions $u: Q \rightarrow \mathbf{R}$, we introduce the following operator

$$
B u \triangleq \beta(x, \tau(t))^{\top} \nabla u(x, \tau(t))+\bar{\beta}(x, \tau(t)) u(x, \tau(t)) .
$$

We assume that the functions $\beta: \mathbf{R}^{n} \times[0, T] \rightarrow \mathbf{R}^{n}$ and $\bar{\beta}: \mathbf{R}^{n} \times[0, T] \rightarrow \mathbf{R}$ are bounded and measurable.

Let us consider the following boundary value problem in $Q$ :

$$
\begin{aligned}
& \frac{\partial u}{\partial t}=\mathcal{A} u+B u+h, \quad t \in(0, T), \\
& u(x, 0)=0,\left.\quad u(x, t)\right|_{x \in \partial D}=0 .
\end{aligned}
$$

Here $\mathcal{A}$ is such as defined above.

Theorem 5 Assume that there exists a constant $\delta_{1}>0$ such that

$$
T\left|\xi^{\top} \beta(x, t)\right|^{2} \leq\left(2-\delta_{1}\right) \xi^{\top} b(x, t) \xi \quad \forall \xi \in \mathbf{R}^{n},(x, t) \in D \times[0, T]
$$

Then there exists a unique solution $u \in Y^{2}$ of problem (9) for any $h \in L_{2}(Q)$, and $\|u\|_{Y^{2}} \leq$ $c\|h\|_{L_{2}(Q)}$, where $c$ is a constant that depends only on $\mathcal{P}, \delta_{1}$, and $\sup _{x, t}|\bar{\beta}(x, t)|$.

Theorem 6 Assume that there exists $\theta \in[0, T)$ such that $\tau(t)=0$ for $t<\theta$. Assume that the function $\tau(\cdot):[\theta, T] \rightarrow \mathbf{R}$ is non-decreasing and absolutely continuous, and $\delta_{*} \triangleq$

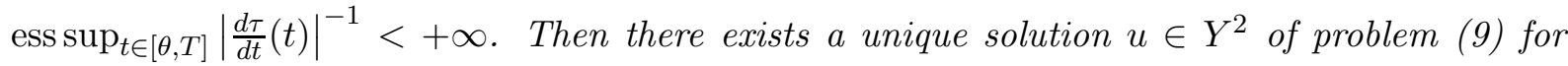
any $h \in L_{2}(Q)$, and $\|u\|_{Y^{2}} \leq c_{*}\|h\|_{L_{2}(Q)}$, where $c_{*}$ is a constant that depends only on $\mathcal{P}, \delta_{*}$, $\sup _{x, t}|\beta(x, t)|$, and $\sup _{x, t}|\bar{\beta}(x, t)|$.

\section{Proofs}

Lemma 1 For any admissible $\mu$ and any $\varepsilon>0, M>0$, there exists $\tilde{K}=\tilde{K}(\varepsilon, M, \mathcal{P}(\mu)) \geq 0$ such that

$$
\begin{array}{r}
(\nabla u(\cdot, t), b(\cdot, t) \nabla u(\cdot, t))_{H^{0}}+M\left(\|u(\cdot, t)\|_{H^{0}}^{2}+\int_{0}^{t}(\nabla u(\cdot, s), b(\cdot, s) \nabla u(\cdot, s))_{H^{0}} d s\right) \\
\leq\left(\frac{1}{2}+\varepsilon\right) \int_{0}^{t}\|h(\cdot, s)\|_{H^{0}}^{2} d s
\end{array}
$$


for all $K \geq \tilde{K}(\varepsilon, M, \mathcal{P}), t \in(0, T]$, and $h \in L_{2}(Q)$, where $u \in Y^{2}$ is the solution of the boundary value problem

$$
\begin{aligned}
& \frac{\partial u}{\partial t}=\mathcal{A} u-K u+h, \quad t \in(0, T), \\
& u(x, 0)=0,\left.\quad u(x, t)\right|_{x \in \partial D}=0 .
\end{aligned}
$$

Uniqueness and existence of solution $u \in Y^{2}$ of problem (12) follows from the classical results (see, e.g., Ladyzhenskaia (1985), Chapter III).

Proof of Lemma 1. Clearly, $\mathcal{A}=\mathcal{A}_{s}+\mathcal{A}_{r}$, where

$$
\mathcal{A}_{s} u=\sum_{i=1}^{n} \frac{\partial}{\partial x_{i}} \sum_{j=1}^{n}\left(b_{i j} \frac{\partial u}{\partial x_{j}}\right)=\nabla \cdot(b \nabla u), \quad \mathcal{A}_{r} u=\sum_{i=1}^{n} f_{i} \frac{\partial u}{\partial x_{i}}+\lambda u .
$$

Assume that the function $h(\cdot, t): D \rightarrow \mathbf{R}$ is differentiable and has a compact support inside $D$ for all $t$. We have that

$$
\begin{aligned}
& (\nabla u(\cdot, t), b(\cdot, t) \nabla u(\cdot, t))_{H^{0}}-(\nabla u(\cdot, 0), b(\cdot, 0) \nabla u(\cdot, 0))_{H^{0}} \\
= & 2 \int_{0}^{t}\left(\nabla u, b \nabla \frac{\partial u}{\partial s}\right)_{H^{0}} d s+\int_{0}^{t}\left(\nabla u, \frac{\partial b}{\partial s} \nabla u\right)_{H^{0}} d s \\
= & 2 \int_{0}^{t}(\nabla u, b \nabla(\mathcal{A} u-K u+h))_{H^{0}} d s+\int_{0}^{t}\left(\nabla u, \frac{\partial b}{\partial s} \nabla u\right)_{H^{0}} d s \\
= & 2 \int_{0}^{t}(\nabla u, b \nabla(\nabla \cdot(b \nabla u)))_{H^{0}} d s+2 \int_{0}^{t}\left(\nabla u, b \nabla \mathcal{A}_{r} u\right)_{H^{0}} d s \\
& -2 K \int_{0}^{t}(\nabla u, b \nabla u)_{H^{0}} d s+2 \int_{0}^{t}(\nabla u, b \nabla h)_{H^{0}} d s+\int_{0}^{t}\left(\nabla u, \frac{\partial b}{\partial s} \nabla u\right)_{H^{0}} d s .
\end{aligned}
$$

Let an arbitrary $\varepsilon_{0}>0$ be given. We have that in under the integrals in (13),

$$
\left(\nabla u, \frac{\partial b}{\partial t} \nabla u\right)_{H^{0}} \leq \operatorname{essup}_{x, t}\left|\frac{\partial b}{\partial t}(x, t)\right|\|\nabla u\|_{H^{0}}^{2} \leq c_{\delta}^{\prime}(\nabla u(\cdot, t), b(\cdot, t) \nabla u(\cdot, t))_{H^{0}},
$$

where $c_{\delta}^{\prime}=c_{\delta}^{\prime}(\mathcal{P})$ is a constant that depends on $\mathcal{P}$ only. Further,

$$
2(\nabla u, b \nabla h)_{H^{0}}=-2(\nabla \cdot(b \nabla u), h)_{H^{0}} \leq \frac{2}{1+2 \varepsilon_{0}}(\nabla u, b \nabla u)_{H^{0}}^{2}+\left(\frac{1}{2}+\varepsilon_{0}\right)\|h\|_{H^{0}}^{2},
$$

Let a bounded measurable function $v(x, t): Q \rightarrow \mathbf{R}$ be such that $b=v^{\top} v$. We have that

$$
2\left(\nabla u, b \nabla \mathcal{A}_{r} u\right)_{H^{0}}=2\left(v \nabla u, v \nabla \mathcal{A}_{r} u\right)_{H^{0}} \leq \varepsilon_{1}^{-1}\|v \nabla u\|_{H^{0}}^{2}+\varepsilon_{1}\left\|v \nabla \mathcal{A}_{r} u\right\|_{H^{0}}^{2} \quad \forall \varepsilon_{1}>0 .
$$

It follows that

$$
2\left(\nabla u, b \nabla \mathcal{A}_{r} u\right)_{H^{0}} \leq \varepsilon_{1}^{-1}(\nabla u, b \nabla u)_{H^{0}}+c_{1} \varepsilon_{1}\|u\|_{H^{2}}^{2}
$$


where $c_{1}=c_{1}(\mathcal{P})$ is a constant that depends on $\mathcal{P}$ only. By the second fundamental inequality, there exists a constant $c_{*}=c_{*}(\mathcal{P})>0$ such that

$$
\int_{0}^{t}\|u(\cdot, s)\|_{H^{2}}^{2} d s \leq c_{*} \int_{0}^{t}\|h(\cdot, s)\|_{H^{0}}^{2} d s .
$$

(See, e.g., estimate (4.56) from Ladyzhenskaia (1985), Chapter III). By Lemma 5.3 from Dokuchaev (2005), one can choose the same constant $c_{*}$ for all $t \in[0, T], K>0$. Hence

$$
2 \int_{0}^{t}\left(\nabla u, b \nabla \mathcal{A}_{r} u\right)_{H^{0}} d s \leq \varepsilon_{1}^{-1} \int_{0}^{t}(\nabla u, b \nabla u)_{H^{0}} d s+\varepsilon_{0} \int_{0}^{t}\|h\|_{H^{0}}^{2} d s
$$

where $\varepsilon_{1}>0$ is such that $c_{1} c_{*} \varepsilon_{1}=\varepsilon_{0}$.

By Lemma 5.2 from Dokuchaev (2005), p. 357, it follows that there exists $K_{1}=K_{1}\left(\varepsilon_{0}, M, \mathcal{P}\right)>$ 0 such that if $K>K_{1}$, then

$$
M \sup _{s \in[0, t]}\|u(\cdot, s)\|_{H^{0}}^{2} \leq \varepsilon_{0} \int_{0}^{t}\|h(\cdot, s)\|_{H^{0}}^{2} d s .
$$

Assume now that $D=\mathbf{R}^{n}$. In that case, we have immediately that

$$
\begin{array}{r}
2(\nabla u, b \nabla(\nabla \cdot(b \nabla u)))_{H^{0}}=2(b \nabla u, \nabla(\nabla \cdot(b \nabla u)))_{H^{0}}=-2(\nabla \cdot(b \nabla u), \nabla \cdot(b \nabla u))_{H^{0}} \\
=-2\|\nabla \cdot(b \nabla u)\|_{H^{0}}^{2} .
\end{array}
$$

The third equality here was obtained using integration by parts.

By (13)-(18), it follows that if $K>K_{1}$ and $2 K>\varepsilon_{1}^{-1}+c_{\delta}^{\prime}+M$, then

$$
\begin{aligned}
& (\nabla u(\cdot, t), b(\cdot, t) \nabla u(\cdot, t))_{H^{0}}+M\left(\|u(\cdot, t)\|_{H^{0}}^{2}+\int_{0}^{t}(\nabla u(\cdot, s), b(\cdot, s) \nabla u(\cdot, s))_{H^{0}} d s\right) \\
& \leq\left(\frac{2}{1+2 \varepsilon_{0}}-2\right) \int_{0}^{t}\|\nabla \cdot(b \nabla u)\|_{H^{0}}^{2} d s+\left(\varepsilon_{1}^{-1}+c_{\delta}^{\prime}+M-2 K\right) \int_{0}^{t}\|v \nabla u\|_{H^{0}}^{2} d s \\
& +\left(\frac{1}{2}+3 \varepsilon_{0}\right) \int_{0}^{t}\|h(\cdot, s)\|_{H^{0}}^{2} d s \leq\left(\frac{1}{2}+3 \varepsilon_{0}\right) \int_{0}^{t}\|h(\cdot, s)\|_{H^{0}}^{2} d s .
\end{aligned}
$$

Then the proof of Lemma 1 follows for the case when $D=\mathbf{R}^{n}$, since (20) holds for all $h$ from a set that is dense in $L_{2}(Q)$.

To complete the proof of Lemma 1, we need to cover the case when $D \neq \mathbf{R}^{n}$. From now and up to the end of the proof of this lemma, we assume that $D \neq \mathbf{R}^{n}$. In that case, (19) does not hold, since the integration by parts used for the third inequality in (19) is not applicable anymore. To replace (19), we are going to show that there exists a constant $C=C(\mathcal{P})>0$ such that, for an arbitrarily $\varepsilon_{2}>0$,

$$
(\nabla u, b \nabla(\nabla \cdot(b \nabla u)))_{H^{0}} \leq-\|\nabla \cdot(b \nabla u)\|_{H^{0}}^{2}+\varepsilon_{2}\|u\|_{H^{2}}^{2}+C \varepsilon_{2}^{-1}\|u\|_{H^{1}}^{2}
$$


Assume that (21) holds. Since $D$ is bounded, we have that $\|u\|_{H^{1}}^{2} \leq c_{\delta}(\nabla u, b \nabla u)_{H^{0}}^{2}$ for some constant $c_{\delta}=c_{\delta}(\mathcal{P})$. In addition, (15) is still valid, since we assumed that $h(\cdot, t)$ have support inside $D$. Similarly to (20), we obtain that if $2 K>\varepsilon_{1}^{-1}+c_{\delta}^{\prime}+C c_{\delta} \varepsilon_{2}^{-1}+M$ and $K>K_{1}$, then

$$
\begin{gathered}
(\nabla u(\cdot, t), b(\cdot, t) \nabla u(\cdot, t))_{H^{0}}+M\left[\|u(\cdot, t)\|_{H^{0}}^{2}+\int_{0}^{t}(\nabla u(\cdot, s), b(\cdot, s) \nabla u(\cdot, s))_{H^{0}} d s\right] \\
\leq\left[\frac{2}{1+2 \varepsilon_{0}}-2\right] \int_{0}^{t}\|\nabla \cdot(b \nabla u)\|_{H^{0}}^{2} d s+\left[\varepsilon_{1}^{-1}+c_{\delta}^{\prime}+M-2 K\right] \int_{0}^{t}(\nabla u(\cdot, s), b(\cdot, s) \nabla u(\cdot, s))_{H^{0}} d s \\
+\left(\frac{1}{2}+3 \varepsilon_{0}\right) \int_{0}^{t}\|h(\cdot, s)\|_{H^{0}}^{2} d s+2 \varepsilon_{2} \int_{0}^{t}\|u(\cdot, s)\|_{H^{2}}^{2} d s+2 C \varepsilon_{2}^{-1} \int_{0}^{t}\|u(\cdot, s)\|_{H^{1}}^{2} d s \\
\leq\left(\frac{1}{2}+3 \varepsilon_{0}+2 c_{*} \varepsilon_{2}\right) \int_{0}^{t}\|h(\cdot, s)\|_{H^{0}}^{2} d s,
\end{gathered}
$$

where $c_{*}$ is the constant from (16). Then the proof of Lemma1 follows provided that (21) holds. Therefore, it suffices to prove (21) for $D \neq \mathbf{R}^{n}$.

Let us prove (21). This estimate can rewritten as

$$
(w, \nabla(\nabla \cdot w))_{H^{0}} \leq-((\nabla \cdot w),(\nabla \cdot w))_{H^{0}}+\varepsilon_{2}\|u\|_{H^{2}}^{2}+C \varepsilon_{2}^{-1}\|u\|_{H^{0}}^{2},
$$

where $w=b \nabla u$. We have

$$
(w, \nabla(\nabla \cdot w))_{H^{0}}=-((\nabla, w),(\nabla \cdot w))_{H^{0}}+\sum_{i=1}^{n} \int_{\partial D} \widehat{J}_{i} d z .
$$

Here $\widehat{J}_{i}=z(\nabla, z) \cos \left(\mathbf{n}, e_{i}\right)$, where $\mathbf{n}=\mathbf{n}(s)$ is the outward pointing normal to the surface $\partial D$ at the point $s \in \partial D$, and $e_{k}=(0, \ldots, 0,1,0, \ldots, 0)$ is the $k$ th basis vector in the Euclidean space $\mathbf{R}^{n}$. We have that

$$
\widehat{J_{i}}=\sum_{j, k, m=1}^{n} \alpha_{i j k m} J_{i j k m}+\sum_{j, k, m=1}^{n} \alpha_{i j k m}^{\prime} J_{i j k m}^{\prime}
$$

where

$$
J_{i j k m}=\frac{\partial u}{\partial x_{j}} \frac{\partial^{2} u}{\partial x_{k} x_{m}} \cos \left(\mathbf{n}, e_{i}\right), \quad J_{i j k}^{\prime}=\frac{\partial u}{\partial x_{j}} \frac{\partial u}{\partial x_{k}} \cos \left(\mathbf{n}, e_{i}\right),
$$

and where $\alpha_{i j k m}, \alpha_{i j k}^{\prime}$ are some bounded functions.

Let us estimate $\int_{\partial D} \widehat{J}_{i} d z$. We mostly follow the approach from Ladyzhenskaya and Ural'tseva (1968), Section 3.8. Let $x^{0}=\left\{x_{i}^{0}\right\}_{i=1}^{n} \in \partial D$ be an arbitrary point. In its neighborhood, we introduce local Cartesian coordinates $y_{m}=\sum_{k=1}^{n} c_{m k}\left(x_{k}-x_{k}^{0}\right)$ such that the axis $y_{n}$ is directed along the outward normal $\mathbf{n}=\mathbf{n}\left(x_{0}\right)$ and $\left\{c_{m k}\right\}$ is an orthogonal matrix.

Let $y_{n}=\psi\left(y_{1}, \ldots, y_{n-1}\right)$ be an equation determining the surface $\partial D$ in a neighborhood of the origin. By the properties of the surface $\partial D$, the first order and second order derivatives of the 
function $\psi$ are bounded. Since $\left\{c_{m k}\right\}$ is an orthogonal matrix, we have $x_{k}-x_{k}^{0}=\sum_{m=1}^{n} c_{k m} y_{m}$. Therefore, $\cos \left(\mathbf{n}, e_{m}\right)=c_{n m}, m=1, \ldots, n$. Then

$$
J_{i j k m}=\sum_{l=1}^{n} c_{j l} \frac{\partial u}{\partial y_{l}} \sum_{p, q=1}^{n} c_{p k} c_{q m} \frac{\partial^{2} u}{\partial y_{p} \partial y_{q}} c_{n i}, \quad J_{i j k}^{\prime}=\sum_{l=1}^{n} c_{j l} \frac{\partial u}{\partial y_{l}} \sum_{p=1}^{n} c_{p k} \frac{\partial u}{\partial y_{p}} c_{n i} .
$$

The boundary condition $\left.u(x, t)\right|_{x \in \partial D}=0$ can be rewritten as

$$
u\left(y_{1}, \ldots, y_{n-1}, \psi\left(y_{1}, \ldots, y^{n-1}\right), t\right)=0
$$

identically with respect to $y_{1}, \ldots, y_{n-1}$ near the point $y_{1}=\ldots=y_{n-1}=0$. Let us differentiate this identity with respect to $y_{p}$ and $y_{q}, p, q=1, \ldots, n-1$, and take into account that

$$
\frac{\partial \psi}{\partial y_{p}}=0, \quad p=1, \ldots, n-1
$$

at $x_{0}$. Then

$$
\frac{\partial u}{\partial y_{p}}=0, \quad \frac{\partial^{2} u}{\partial y_{p} \partial y_{q}}=-\frac{\partial u}{\partial y_{n}} \frac{\partial^{2} \psi}{\partial y_{p} \partial y_{q}}=-\frac{\partial u}{\partial \mathbf{n}} \frac{\partial^{2} \psi}{\partial y_{p} \partial y_{q}}, \quad p, q=1, \ldots, n-1
$$

Hence

$$
\begin{gathered}
\int_{\partial D} \widehat{J}_{i}(z, s) d z=\int_{\partial D} \sum_{j, k, m=1}^{n} \alpha_{i j k m} J_{i j k m}(z, s) d z+\sum_{j, k, m=1}^{n} \int_{\partial D} \alpha_{i j k m}^{\prime} J_{i j k m}^{\prime} \alpha_{i j k m}(z, s) d z \\
\leq \widehat{c}_{1} \int_{\partial D}\left|\frac{\partial u}{\partial \mathbf{n}}\right|^{2} d z \leq \varepsilon_{2} \sum_{i, j=1}^{n} \int_{D}\left|\frac{\partial^{2} u}{\partial x_{i} \partial x_{j}}(x, s)\right|^{2} d x+\widehat{c}_{2}\left(1+\varepsilon_{2}^{-1}\right)\|u(\cdot, s)\|_{H^{1}}^{2} \quad \forall \varepsilon_{2}>0
\end{gathered}
$$

for some constants $\widehat{c}_{i}=\widehat{c}_{i}(\mathcal{P})$. The last estimate follows from the estimate (2.38) from Ladyzhenskaya and Ural'tseva (1968), Chapter II. By (22) and (23), it follows (21). This completes the proof of Lemma 1.

Proof of Theorem 1. Clearly, $u(x, t)=e^{K t} u_{K}(x, t)$, where $u$ is the solution of problem (1) and $u_{K}$ is the solution of (12) for the nonhomogeneous term $e^{-K t} h(x, t)$. Therefore, Theorem 1 follows immediately from Lemma 1

Corollary 1 follows immediately from Theorem 1 ,

Proof of Theorem Q Let $\varepsilon>0$ be given. By Corollary 1, there exists $K(\varepsilon)=K(\varepsilon, \mathcal{P}(\mu))$ such that

$$
\begin{array}{r}
e^{-2 K(\varepsilon) t}(\nabla u(\cdot, t), b(\cdot, t) \nabla u(\cdot, t))_{H^{0}} \leq\left(\frac{1}{2}+\varepsilon\right) \int_{0}^{t} e^{-2 K(\varepsilon) s}\|h(\cdot, s)\|_{H^{0}}^{2} d s \\
\forall t \in(0, T), h \in X^{0} .
\end{array}
$$


Let $p(h, t) \triangleq \frac{1}{t} \int_{0}^{t}\|h(\cdot, s)\|_{H^{0}}^{2} d s$ and $q(u, t) \triangleq(\nabla u(\cdot, t), b(\cdot, t) \nabla u(\cdot, t))_{H^{0}}$. It follows that

$$
\sup _{h \in X^{0}}\left(\frac{q(u, t)}{t p(h, t)}-\frac{1-e^{-2 K(\varepsilon) t}}{t p(h, t)} q(u, t)\right) \leq\left(\frac{1}{2}+\varepsilon\right) \quad \forall t \in(0, T) .
$$

Hence

$$
\sup _{h \in X^{0}} \frac{1}{t p(h, t)} q(u, t) \leq\left(\frac{1}{2}+\varepsilon\right)+\sup _{h \in X^{0}} \frac{1-e^{-2 K(\varepsilon) t}}{t p(h, t)} q(u, t) . \quad \forall t \in(0, T) .
$$

By (24),

$$
q(u, t) \leq e^{2 K(\varepsilon) t}\left(\frac{1}{2}+\varepsilon\right) t p(h, t) \quad \forall t \in(0, T), h \in X^{0}
$$

Hence

$$
\sup _{h \in X^{0}} \frac{1-e^{-2 K(\varepsilon) t}}{t p(h, t)} q(u, t) \rightarrow 0 \quad \text { as } \quad t \rightarrow 0+\quad \forall \varepsilon>0 .
$$

If $h \in X_{c}^{0}$, then $p(h, t) \rightarrow\|h(\cdot, 0)\|_{H^{0}}^{2}$ as $t \rightarrow 0+$. It follows that

$$
\varlimsup_{t \rightarrow 0+} \sup _{h \in X^{c}} \frac{q(u, t)}{t\|h(\cdot, 0)\|_{H^{0}}^{2}} \leq\left(\frac{1}{2}+\varepsilon\right)
$$

for any $\varepsilon>0$. Then (5) follows. This completes the proof of Theorem 2,

Proof of Theorem 3. Repeat that $u(x, t)=e^{K t} u_{K}(x, t)$, where $u$ is the solution of problem (11) and $u_{K}$ is the solution of (12) for $h_{K}(x, t)=e^{-K t} h(x, t)$. Therefore, it suffices to find $n, D$, $b, f, \lambda$ such that

$$
\begin{aligned}
& \forall T>0, c>0, K>0 \quad \exists h \in L_{2}(Q): \\
& (\nabla u(\cdot, T), b(\cdot, T) \nabla u(\cdot, T))_{H^{0}} \geq\left(\frac{1}{2}-c\right) \int_{0}^{T}\|h(\cdot, t)\|_{H^{0}}^{2} d t
\end{aligned}
$$

where $u$ is the solution of problem (12).

Let us show that (25) holds for

$$
n=1, \quad D=(-\pi, \pi), \quad b(x, t) \equiv 1, \quad f(x, t) \equiv 0, \quad \lambda(x, t) \equiv 0 .
$$

In this case, (12) has the form

$$
u_{t}^{\prime}=u_{x x}^{\prime \prime}-K u+h, \quad u(x, 0) \equiv 0,\left.\quad u(x, t)\right|_{x \in \partial D}=0,
$$

Let

$$
\gamma=m^{2}+K, \quad h_{m}(x, t) \triangleq \sin (m x) e^{\gamma t}
$$


where $m=1,2,3, \ldots$. It can be verified immediately that the solution of the boundary value problem is

$$
u(x, t)=\sin (m x) \int_{0}^{t} e^{-\gamma(t-s)+\gamma s} d s=\sin (m x) e^{-\gamma t} \int_{0}^{t} e^{2 \gamma s} d s=\sin (m x) e^{-\gamma t} \frac{e^{2 \gamma t}-1}{2 \gamma} .
$$

Hence

$$
\left\|u_{x}^{\prime}(\cdot, T)\right\|_{H^{0}}^{2}=m^{2}\|\cos (m x)\|_{H^{0}}^{2} e^{-2 \gamma T}\left(\frac{e^{2 \gamma}-1}{2 \gamma}\right)^{2}=m^{2} \pi e^{-2 \gamma T} \frac{\left(e^{2 \gamma T}-1\right)^{2}}{4 \gamma^{2}},
$$

and

$$
\int_{0}^{T}\|h(\cdot, t)\|_{H^{0}}^{2} d t=\|\sin (m x)\|_{H^{0}}^{2} \int_{0}^{T} e^{2 \gamma t} d t=\pi \frac{e^{2 \gamma T}-1}{2 \gamma} .
$$

It follows that

$$
\left\|u_{x}^{\prime}(\cdot, T)\right\|_{H^{0}}^{2}\left(\int_{0}^{T}\|h(\cdot, t)\|_{H^{0}}^{2} d t\right)^{-1}=\frac{m^{2}}{2 \gamma} e^{-2 \gamma T}\left(e^{2 \gamma T}-1\right)=\frac{m^{2}}{2 \gamma}\left(1-e^{-2 \gamma T}\right) \rightarrow \frac{1}{2}
$$

as $\gamma \rightarrow+\infty$. In particular, it holds if $K$ is fixed and $m \rightarrow+\infty$. It follows that (16) holds. This completes the proof of Theorem 3 .

Proof of Theorem 4. Let the parameters of the equation be defined by (26). Consider a sequence $\left\{T_{i}\right\}$ such that $T_{i} \rightarrow 0+$ as $i \rightarrow+\infty$. Let $h=h_{m}$ be defined by (28) for an increasing sequence of integers $m=m_{i}$ such that $m_{i}>T_{i}^{-1}$. In that case, (29) holds since $\gamma T \rightarrow+\infty$. Then (77) holds.

Proof of Theorem [5. For $K>0$, introduce operators

$$
B_{K} u \triangleq e^{K(\tau(t)-t)}\left(\beta(x, \tau(t))^{\top} \nabla u(x, \tau(t))+\bar{\beta}(x, \tau(t)) u(x, \tau(t))\right) .
$$

Note that $u \in Y^{2}$ is the solution of the problem (9) if and only if $u_{K}(x, t)=e^{-K t} u(x, t)$ is the solution of the problem

$$
\begin{aligned}
& \frac{\partial u_{K}}{\partial t}=\mathcal{A} u_{K}-K u_{K}+B_{K} u_{K}+h_{K}, \quad t \in(0, T), \\
& u_{K}(x, 0)=0,\left.\quad u_{K}(x, t)\right|_{x \in \partial D}=0,
\end{aligned}
$$

where $h_{K}(x, t)=e^{-K t} h(x, t)$. In addition,

$$
\|u\|_{Y^{2}} \leq e^{K T}\left\|u_{K}\right\|_{Y_{2}}, \quad\left\|h_{K}\right\|_{L_{2}(Q)} \leq\|h\|_{L_{2}(Q)}
$$

Therefore, uniqueness and solvability in $Y^{2}$ of problem (9) follows from existence of $K>0$ such that problem (31) has an unique solution in $Y^{2}$. Let us show that this $K$ can be found. 
We introduce operators $F_{K}: L_{2}(Q) \rightarrow Y^{2}$ such that $u=F_{K} h$ is the solution of problem (12).

Let $g \in L_{2}(Q)$ be such that

$$
g=h+B_{K} w, \quad \text { where } \quad w=F_{K} g .
$$

It can be rewritten as $g=h+R_{K} g$, or

$$
g-R_{K} g=h
$$

where the operator $R_{K}: L_{2}(Q) \rightarrow L_{2}(Q)$ is defined as

$$
R_{K}=B_{K} F_{K}
$$

In that case, $u_{K} \triangleq F_{K} g \in Y^{2}$ is the solution of (31).

Let us show that there exists $K>0$ such that

$$
\left\|R_{K}\right\|<1
$$

Let $w=F_{K} h$. By Theorem 1 reformulated as Lemma 1, for any $\varepsilon>0, M>0$, there exists $K(\varepsilon, M, \mathcal{P}(\mu)) \geq 0$ such that

$$
\begin{gathered}
\sup _{t \in[0, T]}\left((\nabla w(\cdot, t), b(\cdot, t) \nabla w(\cdot, t))_{H^{0}}+M\|w(\cdot, t)\|_{H^{0}}^{2}+M \int_{0}^{t}(\nabla w(\cdot, s), b(\cdot, s) \nabla w(\cdot, s))_{H^{0}} d s\right) \\
\leq\left(\frac{1}{2}+\varepsilon\right)\|h\|_{L_{2}(Q)}^{2} d s \quad \forall h \in L_{2}(Q) .
\end{gathered}
$$

Let $\widehat{w}(x, t) \triangleq w(x, \tau(t)), \widehat{\beta}(x, t) \triangleq \beta(x, \tau(t))$, and $\widehat{b}(x, t) \triangleq b(x, \tau(t))$. By the definitions,

$$
\left\|R_{K} h\right\|_{L_{2}(Q)}=\left\|B_{K} w_{K}\right\|_{L_{2}(Q)} \leq\left\|\widehat{\beta}^{\top} \nabla \widehat{w}\right\|_{L_{2}(Q)}+C_{\beta}\|\widehat{w}\|_{L_{2}(Q)},
$$

where $C_{\beta} \triangleq \sup _{x, t}|\bar{\beta}(x, t)|$. Clearly,

$$
\begin{aligned}
& \left\|\widehat{\beta}^{\top} \nabla \widehat{w}\right\|_{L_{2}(Q)}^{2} \leq T \sup _{t \in[0, T]}\left\|\widehat{\beta}(\cdot, t)^{\top} \nabla \widehat{w}(\cdot, t)\right\|_{H^{0}}^{2} \leq T \sup _{t \in[0, T]}\left\|\beta(\cdot, t)^{\top} \nabla w(\cdot, t)\right\|_{H^{0}}^{2}, \\
& \|\widehat{w}\|_{L_{2}(Q)}^{2} \leq T \sup _{t \in[0, T]}\|\widehat{w}(\cdot, t)\|_{H^{0}}^{2} \leq T \sup _{t \in[0, T]}\|w(\cdot, t)\|_{H^{0}}^{2} .
\end{aligned}
$$

Further, let $K>0, M>0$, and $\varepsilon>0$, be such that (35) is satisfied and

$$
\delta_{2}<1, \quad \delta_{2}^{2}+\delta_{3}^{2}+2 \delta_{2} \delta_{3}<1
$$

where

$$
\delta_{2} \triangleq \sqrt{\left(2-\delta_{1}\right)\left(\frac{1}{2}+\varepsilon\right)}, \quad \delta_{3} \triangleq C_{\beta} \sqrt{T M^{-1}\left(\frac{1}{2}+\varepsilon\right)}
$$


By (35), (37)-(40), and (10), it follows that

$$
\left\|\widehat{\beta}^{\top} \nabla \widehat{w}\right\|_{L_{2}(Q)}^{2} \leq T \sup _{t \in[0, T]}\left\|\beta(\cdot, t)^{\top} \nabla w(\cdot, t)\right\|_{H^{0}}^{2} \leq\left(2-\delta_{1}\right) \sup _{t \in[0, T]}(\nabla w, b \nabla w)_{H^{0}} \leq \delta_{2}^{2}\|h\|_{L_{2}(Q)}^{2} .
$$

In addition, we have that

$$
C_{\beta}^{2}\|\widehat{w}\|_{L_{2}(Q)}^{2} \leq T C_{\beta}^{2} \sup _{t \in[0, T]}\|w(\cdot, t)\|_{H^{0}}^{2} \leq T C_{\beta}^{2} M^{-1}\left(\frac{1}{2}+\varepsilon\right)\|h\|_{L_{2}(Q)}^{2} d s \leq \delta_{3}^{2}\|h\|_{L_{2}(Q)}^{2}
$$

By (36), it follows that

$$
\left\|R_{K} h\right\|_{L_{2}(Q)}^{2} \leq\left(\left\|\widehat{\beta}^{\top} \nabla \widehat{w}\right\|_{L_{2}(Q)}+C_{\beta}\|\widehat{w}\|_{L_{2}(Q)}\right)^{2} \leq\left(\delta_{2}^{2}+2 \delta_{2} \delta_{3}+\delta_{3}^{2}\right)\|h\|_{L_{2}(Q)}^{2} .
$$

By (39), it follows that (34) holds, where the norm of the operator $R_{K}: L_{2}(Q) \rightarrow L_{2}(Q)$ is considered. It follows that the operator $\left(I-R_{K}\right)^{-1}: L_{2}(Q) \rightarrow L_{2}(Q)$ is continuous, By (32)-(33), $u_{K}=F_{K}\left(I-R_{K}\right)^{-1} h_{K}$ is the solution of problem (31) for $h_{K} \in L_{2}(Q)$.

The choice of $K, M$, and $\varepsilon$, depends on $\mathcal{P}, \delta_{1}$, and $\sup _{x, t}|\bar{\beta}(x, t)|$ only. Hence $\delta_{2}$ and $\delta_{3}$ depends on these parameters only. It follows that the norm of the operator $\left(I-R_{K}\right)^{-1}: L_{2}(Q) \rightarrow$ $L_{2}(Q)$ can be estimated from above by a constant that depends only on these parameters. This proves the estimate for the solution stated in Theorem 5. This completes the proof of this theorem.

Proof of Theorem 6 is based again on (35). It is similar to the proof of Theorem 5 , with a minor modification: instead of (37), we use that

$$
\begin{aligned}
& \int_{0}^{T}\left\|\beta(\cdot, \tau(t))^{\top} \nabla u(\cdot, \tau(t))\right\|_{H^{0}}^{2} d t=\int_{\theta}^{T}\left\|\beta(\cdot, \tau(t))^{\top} \nabla u(\cdot, \tau(t))\right\|_{H^{0}}^{2} d t \\
& =\int_{\theta}^{T}\left\|\beta(\cdot, \tau(t))^{\top} \nabla u(\cdot, \tau(t))\right\|_{H^{0}}^{2}\left(\frac{d \tau(t)}{d t}\right)^{-1} d \tau(t) \leq \delta_{*} \int_{\tau(\theta)}^{\tau(T)}\left\|\beta(\cdot, s)^{\top} \nabla u(\cdot, s)\right\|_{H^{0}}^{2} d s \\
& \leq c \int_{0}^{T}(\nabla u(\cdot, s), b(\cdot, s) \nabla u(\cdot, s))_{H^{0}} d s
\end{aligned}
$$

where $c$ is a constant that depends on $\delta, \delta_{*}$, and $\sup _{x, t}|\beta(x, t)|$.

It can be seen from the proofs that the approach used for Theorems 56 can be extended on more general delay operators represented by integrals accumulating the past values.

\section{Acknowledgment}

This work was supported by NSERC grant of Canada 341796-2008 to the author. 


\section{References}

Bátkai, A., Piazzera, A.S. (2001). Semigroups and linear partial differential equations with delay. J. Math. Anal. Appl. 264, 1-20.

Dokuchaev, N.G. (2005). Parabolic Ito equations and second fundamental inequality. Stochastics 77, iss. 4., 349-370.

Ladyzhenskaya, O. A., and Ural'tseva, N.N. (1968). Linear and quasilinear elliptic equations. New York: Academic Press.

Ladyzhenskaia, O.A. (1985). The Boundary Value Problems of Mathematical Physics. New York: Springer-Verlag.

Pao, C.V. (1997). System of Parabolic Equations with Continuous and Discrete Delays, J. Math. Anal. Appl., 205, 157-185.

Poorkarimi, C.H., Wiener, J. (1999). Bounded solutions of nonlinear parabolic equations with time delay. Electronic J. Differential Equations, 1999, pp. 87-91.

Stein, M., Vogt H., Vöigt, J. (2005). The modulus semigroup for linear delay equations III. J. Funct. Anal., 220 (2), 388-400. 